\title{
Fatores de risco, proteção, diagnóstico e tratamento da depressão pós-parto no contexto da atenção primária
}

Risk factors, protection, diagnostic and treatment of postpartum depression at the primary care's context

Factores de riesgo, protección, diagnóstico y tratamiento de la depresión pós-parto en el contexto de la atención primaria de salud

Camila Azalim de Campos ${ }^{1 *}$, Hérica Maris Martins Silva ${ }^{1}$, Mariana Melo Franco Viviani ${ }^{1}$, Matheus Moraes Alves Pereira ${ }^{1}$, Renata Costa Santos ${ }^{1}$, Sílvia Eutrópio Vasconcelos ${ }^{1}$, Thauan Pedro da Silva Santos ${ }^{1}$, Victor Eliel Bastos de Carvalho ${ }^{1}$, Victória Cristine Araujo Dias ${ }^{1}$, Eduardo Siqueira Fernandes ${ }^{1}$.

\section{RESUMO}

Objetivo: Buscar informações acerca dos fatores de risco e de proteção para a DPP, formas de diagnóstico e tratamentos, indicando, assim, os níveis de evidência nas pesquisas. Métodos: Trata-se de uma revisão bibliográfica baseada em estudos publicados de 2015-2020, nas bases de dados PubMed, Literatura latinoamericana e do caribe em ciências da saúde (LILACS) e Scientific Electronic Library Online (Scielo). Resultados: Foram identificados 96 estudos, desses, 12 foram selecionados com base nos critérios de inclusão e exclusão do presente estudo. A maioria dos estudos evidenciou como principais fatores de risco o baixo nível socioeconômico, o histórico de depressão e a falta de apoio social à mulher. O principal fator de proteção foi o acompanhamento da gestante por uma equipe multidisciplinar. Os métodos de diagnóstico para a DPP citados foram a Escala de Depressão Pós-parto de Edimburgo (7 estudos), a Depression Anxiety Stress Scales (DASS-42) (1 estudo). As formas de tratamento citadas são: terapia psicossocial, tratamento medicamentoso, técnicas de entrevistas motivacionais e terapia cognitiva comportamental. Considerações Finais: A baixa condição socioeconômica, o baixo grau de escolaridade e a violência doméstica são os fatores de risco mais prevalentes e, portanto, é essencial pensar em ações sociais e de saúde que revertam esse contexto.

Palavras-chave: Depressão pós-parto, Fatores de risco, Atenção primária à saúde.

\begin{abstract}
Objective: To seek information about risk and protective factors for PPD, forms of diagnosis and treatments, thus indicating the levels of evidence in the research. Methods: This is a bibliographic review based on studies published in 2015-2020, in the databases PubMed, Latin American and Caribbean Literature in Health Sciences (LILACS) and Scientific Electronic Library Online (Scielo). Results: 96 studies were identified, of these, 12 were selected based on the inclusion and exclusion criteria of the present study. Most studies have found low socioeconomic status, history of depression and lack of social support for women as the main risk factors. The main protective factor was the monitoring of the pregnant woman by a multidisciplinary team. The diagnostic methods for PPD mentioned were the Edinburgh Postpartum Depression Scale (7 studies), the Depression Anxiety Stress Scales (DASS-42) (1 study). The forms of treatment mentioned are: psychosocial therapy, drug treatment, motivational interviewing techniques and cognitive behavioral therapy. Final Considerations: Low socioeconomic status, low level of education and domestic violence are the most prevalent risk factors and, therefore, it is essential to think about social and health actions that reverse this context.
\end{abstract}

Keywords: Postpartum depression, Risk factors, Primary care.

${ }^{1}$ Pontifícia Universidade Católica de Minas Gerais (PUC-MG), Betim - MG. *E-mail: azalimcamila@gmail.com

PUBLICADO EM: 1/2021 


\section{RESUMEN}

Objetivo: Buscar información sobre factores de riesgo y protectores para la DPP, formas de diagnóstico y tratamientos, indicando así los niveles de evidencia en la investigación. Métodos: Se trata de una revisión bibliográfica basada en estudios publicados en 2015-2020, en las bases de datos PubMed, Literatura Latinoamericana y del Caribe en Ciencias de la Salud (LILACS) y Scientific Electronic Library Online (Scielo). Resultados: Se identificaron 96 estudios, de los cuales 12 fueron seleccionados con base en los criterios de inclusión y exclusión del presente estudio. La mayoría de los estudios han encontrado un nivel socioeconómico bajo, antecedentes de depresión y falta de apoyo social para las mujeres como los principales factores de riesgo. El principal factor protector fue el seguimiento de la gestante por un equipo multidisciplinario. Los métodos de diagnóstico de la depresión posparto mencionados fueron la Escala de depresión posparto de Edimburgo (7 estudios), las Escalas de estrés, ansiedad y depresión (DASS-42) (1 estudio). Las formas de tratamiento mencionadas son: terapia psicosocial, tratamiento farmacológico, técnicas de entrevista motivacional y terapia cognitivo-conductual. Consideraciones Finales: El bajo nivel socioeconómico, el bajo nivel educativo y la violencia intrafamiliar son los factores de riesgo más prevalentes $y$, por lo tanto, es fundamental pensar en acciones sociales y de salud que reviertan este contexto.

Palabras clave: Depresión posparto, Factores de riesgo, Atención primaria de salud.

\section{INTRODUÇÃO}

A depressão pós-parto (DPP) é um grave problema de saúde pública que se dá a partir de um episódio de depressão maior temporalmente associado ao nascimento de um bebê (APA, 1994). Segundo o Manual Diagnóstico e Estatístico de Transtornos Mentais, da Sociedade Americana de Psiquiatria, a sintomatologia aparece até as 4 semanas pós-parto (DSM-5, 2014).

A 11ª revisão da Classificação Internacional de Doenças (2020), contudo, caracteriza que a DPP pode surgir até 6 semanas pós-parto. Alguns autores defendem que seus sintomas podem aparecer ainda durante a gravidez, em seu último mês, até cinco meses após o parto (ARRAIAS AR e ARAUJO TCCF, 2017). Dessa forma, ainda existem divergências na conceituação de DPP na literatura, e por isso muitos autores ao se referirem a episódios de depressão maior associados à maternidade trocam o termo para depressão perinatal.

Diversos trabalhos apontam também que a depressão perinatal pode resultar em efeitos negativos a curto e a longo prazo na mulher e na criança (O'CONNOR E, et al., 2019). Os sintomas mais comuns da DPP incluem perda de interesse e energia, humor deprimido, flutuações nos padrões de sono ou alimentação, capacidade reduzida de pensar ou se concentrar, sentimentos de inutilidade e ideação suicida recorrente. É importante ressaltar que para o diagnóstico de DPP é necessário que a mulher apresente obrigatoriamente humor deprimido e/ou perda de interesse por um período mínimo de duas semanas (AMERICAN COLLEGE OF OBSTETRICIANS AND GYNECOLOGISTS, 2015).

Associadas a esse quadro clínico podem surgir queixas diversas, não necessariamente correlacionadas à distúrbios de humor, como cefaleia, hemorragias, dificuldades na lactação devido a presença de fissuras e infecções, entre outras (MELO SB, et al., 2018).

A prevalência da depressão pós-parto nos países desenvolvidos varia entre 5,2 e 74\% (FERREIRA C, et al., 2018), a depender da região ou do instrumento diagnóstico utilizado. Além disso, a presença de sintomas depressivos afeta uma em cada sete mulheres durante a gravidez ou após o parto, e é uma das complicações mais comuns da gravidez e do período pós-parto (FORÇA-TAREFA DE SERVIÇOS PREVENTIVOS DOS EUA, 2019).

O hospital das clínicas de São Paulo foi, no Brasil, o primeiro a evidenciar a importância de se atentar à existência da depressão pós-parto ao abrir, na década de 90 , um serviço voltado para essas mulheres. Ainda recentemente, os estudos têm evidenciado que a sintomatologia pode surgir em torno de seis a 18 meses após o parto em $25 \%$ das mulheres brasileiras (FILHA T, et al., 2016). 
A gestação e o pós-parto são processos que provocam transformações na vida das mulheres, incluindo mudanças sociais, econômicas, laborais e principalmente fisiológicas. Além disso, existe socialmente uma imagem romantizada da mulher-mãe como feliz e realizada, como se o tornar-se mãe fizesse parte da natureza e essência da mulher (MELO SB, et al., 2018).

O momento após o parto é carregado de emoções fortes e de novos desafios no processo de tornar-se mãe. Nesta perspectiva, a mulher vive cercada de cobranças e idealizações sobre o seu papel de mãe e, por vezes, não dispõe de um adequado suporte social que a auxilie a desempenhar essa atribuição (MELO SB, et al., 2018).

Dessa maneira, torna-se importante identificar mulheres nesse ciclo gravídico-puerperal, para evitar a piora da depressão (KO JY, et al. 2017) e também possíveis complicações para o recém-nascido, de acordo com Xu F, et al. (2018). Considerando que a saúde materna é sensível à Atenção Primária à Saúde (APS), é possível acompanhar a mulher em todas as fases do pré-natal ao puerpério, sendo estes, momentos-chave para políticas públicas e diretrizes clínicas da Saúde da Mulher (BARATIERI T e NATAL S, 2018).

O presente artigo tem como objetivo reunir informações da literatura científica produzida sobre os fatores de risco e de proteção para a DPP, suas formas de diagnóstico e de tratamentos, indicando, assim, os níveis de evidência nas pesquisas.

\section{MÉTODOS}

Após a delimitação do tema de escolha para o artigo, foi utilizado o site da Biblioteca Virtual de Saúde (BVS), para a pesquisa dos Descritores em Ciência da Saúde (DeCS). A partir dessa consulta ao DeCS, foram escolhidos os seguintes descritores: "Post partum Depression", "Risk Factors" e "PrimaryCare", e suas respectivas traduções em português e espanhol.

Na sequência foram utilizadas três bases de dados para a pesquisa de artigos: PubMed, LILACS e Scielo. Os termos foram inseridos em formulário avançado, combinados entre si. No PubMed, os descritores utilizados para a pesquisa, resultaram em 93 artigos. Subsequentemente, foi adicionado o filtro para tempo, selecionando artigos com menos de 5 anos e o filtro para espécies humanas, o que reduziu o número de artigos para 75 .

$\mathrm{Na}$ base de dados LILACS, os descritores pesquisados resultaram em três artigos, destes, foram escolhidos dois por abordarem a espécie humana. Na terceira base de dados utilizada, o Scielo, foram utilizados os mesmos descritores supracitados, resultando em zero artigos.

\section{RESULTADOS}

A pesquisa nas três bases de dados, Pubmed, LILACS e Scielo, trouxe, no total, 77 artigos. Os artigos foram inicialmente repartidos para cada pesquisador (aproximadamente nove artigos para cada) e avaliados quanto ao título e resumo, com objetivo de identificar correlação ao tema proposto. Foram excluídos nessa análise artigos cujos desenhos de estudo não fossem do tipo observacional (Coorte, Caso-controle e Transversal) ou Ensaios Clínicos - 27 artigos seguiram na seleção.

A partir da amostra de 27 artigos, três pesquisadores continuaram a seleção. Foram utilizados como fatores de inclusão: (1) Artigos com metodologia bem delimitada e descrita; (2) Estudos disponíveis na íntegra; (3) Estudos que incluíssem o período de puerpério nas análises. Doze artigos então restaram para análise final (Figura 1). 
Figura 1 - Metodologia de escolha dos artigos.

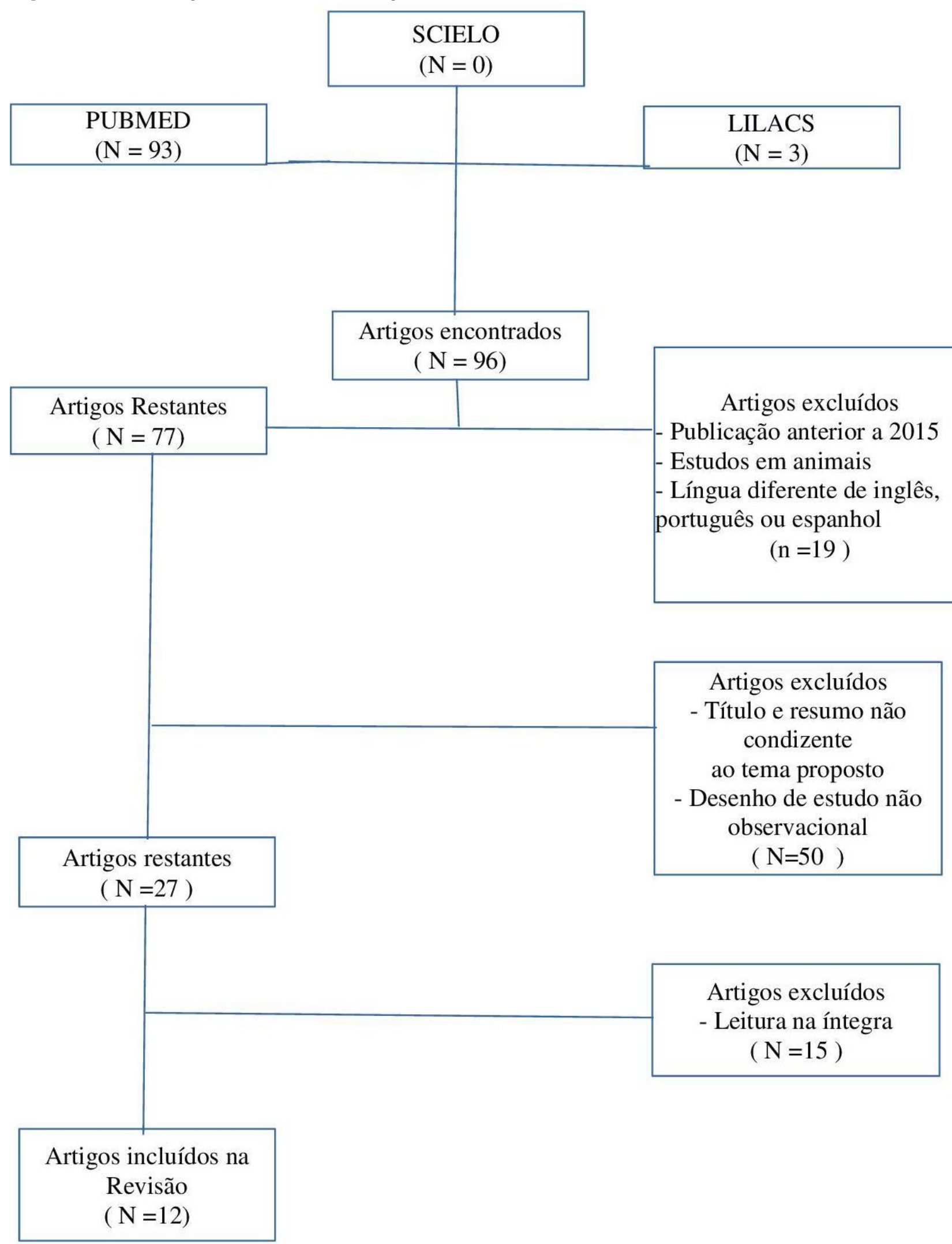

Fonte: Campos CA, et al., 2020.

A partir da análise dos 12 artigos selecionados na metodologia foi possível compreender melhor os fatores de risco e proteção (Quadro 1) e diagnóstico e tratamento da DPP (Quadro 2). 


\section{Revista Eletrônica Acervo Saúde / Electronic Journal Collection Health | ISSN 2178-2091}

Quadro 1 - Fatores de risco e proteção encontrados nos estudos selecionados.

\begin{tabular}{|c|c|c|c|c|}
\hline $\begin{array}{l}\text { Autores / Ano de } \\
\text { publicação }\end{array}$ & $\begin{array}{c}\text { Desenho do estudo / } \\
\text { Amostra (N) }\end{array}$ & Local do estudo & Fatores de risco encontrados & $\begin{array}{l}\text { Fatores de proteção } \\
\text { encontrados }\end{array}$ \\
\hline $\begin{array}{l}\text { OZTORA S, et } \\
\text { al., } 2019\end{array}$ & $\begin{array}{c}\text { Estudo de Coorte } \\
\text { Prospectivo / N=111 }\end{array}$ & $\begin{array}{l}\text { Serviços de atenção } \\
\text { primária na cidade de } \\
\text { Edirne, Turquia. }\end{array}$ & $\begin{array}{l}\text { - Maternidade em mulheres menores de } 18 \text { anos } \\
\text { - Cônjuge estar desempregado durante gestação } \\
\text { - Baixo nível socioeconômico } \\
\text { - O bebê ter problemas de saúde }\end{array}$ & $\begin{array}{l}\text { - Idade materna maior de } 18 \\
\text { anos } \\
\text { - Amamentação regular }\end{array}$ \\
\hline $\begin{array}{l}\text { WASSIF OM, et } \\
\text { al., } 2019\end{array}$ & $\begin{array}{l}\text { Estudo Observacional } \\
\text { Transversal / N=500 }\end{array}$ & $\begin{array}{c}\text { Unidades básicas de } \\
\text { saúde na província de } \\
\text { Qaliubeya, Egito }\end{array}$ & $\begin{array}{l}\text { - Nível socioeconômico baixo } \\
\text { - Níveis educacionais baixos } \\
\text { - Baixo nível de progesterona } \\
\text { - Histórico anterior de depressão }\end{array}$ & $\begin{array}{l}\text { - Aconselhamento } \\
\text { - Grupos de apoio } \\
\text { - Estratégias de autoajuda }\end{array}$ \\
\hline $\begin{array}{l}\text { MA JH, et al., } \\
2019\end{array}$ & $\begin{array}{c}\text { Estudo de Ensaio } \\
\text { Clínico duplo-cego } \\
\text { Randomizado / N=702 }\end{array}$ & $\begin{array}{l}\text { ThirdXiangya Hospital of } \\
\text { Central South } \\
\text { University, Changsha, } \\
\text { China }\end{array}$ & - Histórico anterior de depressão & $\begin{array}{l}\text { - Uso de quetamina } \\
\text { profilática e pacientes com } \\
\text { risco de desenvolver } \\
\text { depressão }\end{array}$ \\
\hline $\begin{array}{l}\text { SHAKEEL N, et } \\
\text { al., } 2018\end{array}$ & $\begin{array}{c}\text { Estudo de Coorte } \\
\text { Prospectiva / N=643 }\end{array}$ & $\begin{array}{l}\text { Clínicas para } \\
\text { atendimento pré-natal } \\
\text { em Groruddalen, Oslo, } \\
\text { Noruega }\end{array}$ & $\begin{array}{l}\text { - Eventos adversos recentes da vida } \\
\text { - Falta de apoio social } \\
\text { - Sintomas depressivos no início da gravidez } \\
\text { - Minoria étnica } \\
\text { - Baixa integração social. }\end{array}$ & Não relata \\
\hline $\begin{array}{l}\text { MELTZER- } \\
\text { BRODY S, et al., } \\
2018\end{array}$ & $\begin{array}{c}\text { Estudo de Coorte } \\
\text { Prospectiva / } \mathrm{N}=85080\end{array}$ & Dinamarca & $\begin{array}{l}\text { Adversidades na infância: } \\
\text {-Ter sido colocada para fora de casa } \\
\text {-Psicopatologia parental excluindo abuso de } \\
\text { substâncias } \\
\text {-Perda de emprego pelos pais na infância } \\
\text {-Doenças somáticas dos pais } \\
\text {-Deficiências dos pais }\end{array}$ & Não relata \\
\hline
\end{tabular}

REAS/EJCH | Vol.13(1) | e5410 | DOI: https://doi.org/10.25248/reas.e5410.2021 


\section{Revista Eletrônica Acervo Saúde / Electronic Journal Collection Health | ISSN 2178-2091}

\begin{tabular}{|c|c|c|c|c|}
\hline $\begin{array}{l}\text { Autores / Ano de } \\
\text { publicação }\end{array}$ & $\begin{array}{c}\text { Desenho do estudo / } \\
\text { Amostra (N) }\end{array}$ & Local do estudo & Fatores de risco encontrados & $\begin{array}{l}\text { Fatores de proteção } \\
\text { encontrados }\end{array}$ \\
\hline $\begin{array}{l}\text { HOLT C, et al., } \\
\qquad 2017\end{array}$ & $\begin{array}{c}\text { Estudo de Ensaio } \\
\text { Clínico duplo-cego } \\
\text { Randomizado / N=541 }\end{array}$ & Victoria, Austrália & $\begin{array}{l}\text { Não foram encontrados fatores estatisticamente } \\
\text { significativos }\end{array}$ & $\begin{array}{l}\text { Não foram encontrados } \\
\text { fatores estatisticamente } \\
\text { significativos }\end{array}$ \\
\hline XU F, et al., 2018 & $\begin{array}{l}\text { Estudo de Coorte } \\
\text { Prospectiva / } \mathrm{N}= \\
345049\end{array}$ & $\begin{array}{c}\text { New South } \\
\text { Wales, Austrália }\end{array}$ & - Anemia -Estresse & $\begin{array}{l}\text { - Identificação e tratamento } \\
\text { precoce das pacientes } \\
\text { anêmicas }\end{array}$ \\
\hline $\begin{array}{l}\text { TABB KM, et al., } \\
2018\end{array}$ & $\begin{array}{l}\text { Estudo Observacional } \\
\text { Transversal / } \mathrm{N}=701\end{array}$ & $\begin{array}{c}\text { Clínicas de atenção } \\
\text { primária em São Paulo }\end{array}$ & $\begin{array}{l}\text { - Violência doméstica } \\
\text { - Baixa renda } \\
\text { - Ideação suicida }\end{array}$ & - Rede de apoio \\
\hline $\begin{array}{l}\text { MUNK-OLSEN T, } \\
\text { et al., } 2015\end{array}$ & $\begin{array}{c}\text { Estudo de Coorte } \\
\text { Prospectiva / } \mathrm{N}=6630\end{array}$ & Dinamarca & $\begin{array}{l}\text { - Transtorno Afetivo Bipolar } \\
\text { - Saúde mental previamente vulnerável }\end{array}$ & $\begin{array}{l}\text { - Identificação precoce de } \\
\text { sinais depressivos } \\
\text { - Acompanhamento } \\
\text { longitudinal }\end{array}$ \\
\hline $\begin{array}{l}\text { ROGATHI JJ, et } \\
\text { al., } 2017\end{array}$ & $\begin{array}{c}\text { Estudo de Coorte } \\
\text { Prospectiva / } \mathrm{N}=1303\end{array}$ & $\begin{array}{c}\text { Clínicas pré-natais na } \\
\text { região de Kilimanjaro, } \\
\text { Tanzânia }\end{array}$ & $\begin{array}{l}\text { - Violência doméstica } \\
\text { - Países de baixa renda } \\
\text { - Mulheres mais jovens }\end{array}$ & $\begin{array}{l}\text { - Mulheres mais velhas } \\
\text { - Identificação precoce de } \\
\text { sinais depressivos }\end{array}$ \\
\hline $\begin{array}{l}\text { NGAI FW, et al., } \\
2017\end{array}$ & $\begin{array}{l}\text { Estudo de Ensaio } \\
\text { Clínico Randomizado/ } \\
\qquad \mathrm{N}=1605\end{array}$ & $\begin{array}{l}\text { Hospitais públicos em } \\
\text { Hong Kong }\end{array}$ & - Não relata & $\begin{array}{l}\text { - Terapia cognitivo } \\
\text { comportamental }\end{array}$ \\
\hline $\begin{array}{l}\text { GIALLO R, et al., } \\
\qquad 2015\end{array}$ & $\begin{array}{c}\text { Estudo de Coorte } \\
\text { Prospectiva / } \mathrm{N}=1459\end{array}$ & $\begin{array}{l}\text { La TrobeUniversity, e o } \\
\text { Royal Children's } \\
\text { Hospital, na Australia }\end{array}$ & - Fadiga & $\begin{array}{l}\text { - Prevenção e controle da } \\
\text { fadiga }\end{array}$ \\
\hline
\end{tabular}

Fonte: Campos CA, et al., 2020. 


\section{Revista Eletrônica Acervo Saúde / Electronic Journal Collection Health | ISSN 2178-2091}

Quadro 2 - Diagnóstico e tratamento encontrados nos artigos selecionados.

\begin{tabular}{|c|c|c|}
\hline Autores / Ano de publicação & Diagnóstico & Tratamento \\
\hline OZTORA S, et al., 2019 & Escala de Depressão Pós-parto de Edimburgo (ponto de corte $\geq 11$ ) & Não cita formas de tratamento \\
\hline WASSIF OM, et al., 2019 & DepressionAnxiety Stress Scales (DASS-42) & Não cita formas de tratamento \\
\hline MA JH, et al., 2019 & Escala de Depressão Pós-parto de Edimburgo (ponto de corte > 9) & $\begin{array}{l}\text { - Terapia psicossocial } \\
\text { - Tratamento medicamentoso }\end{array}$ \\
\hline SHAKEEL N, et al., 2018 & Escala de Depressão Pós-parto de Edimburgo (ponto de corte $\geq 10$ ) & Não cita formas de tratamento \\
\hline MELTZER-BRODY S, et al., 2018 & Não cita formas de diagnóstico & Não cita formas de tratamento \\
\hline HOLT C, et al., 2017 & $\begin{array}{l}\text {-Escala de Depressão Pós-parto de Edimburgo (ponto de corte } \geq 13 \text { ) } \\
\text {-Depressionanxiety stress scales-Short form (DASS21) }\end{array}$ & Técnica de entrevistas motivacionais \\
\hline XU F, et al., 2018 & Não cita formas de diagnóstico & Não cita formas de tratamento \\
\hline TABB KM, et al., 2018 & Não cita formas de diagnóstico & Não cita formas de tratamento \\
\hline MUNK-OLSEN T, et al., 2015 & Não cita formas de diagnóstico & Não cita formas de tratamento \\
\hline ROGATHI JJ, et al., 2017 & Escala de Depressão Pós-parto de Edimburgo (ponto de corte $\geq 13$ ) & Não cita formas de tratamento \\
\hline NGAI FW, et al., 2017 & Escala de Depressão Pós-parto de Edimburgo (ponto de corte 9/10) & Terapia Cognitiva Comportamental \\
\hline GIALLO R, et al., 2015 & Escala de Depressão Pós-parto de Edimburgo (ponto de corte $\geq 13$ ) & Não cita formas de tratamento \\
\hline
\end{tabular}

Fonte: Campos CA, et al., 2020.

$$
\text { REAS/EJCH | Vol.13(1) | e5410 | DOI: https://doi.org/10.25248/reas.e5410.2021 Página } 7 \text { de } 11
$$




\section{DISCUSSÃO}

A maioria dos estudos encontrados evidenciou como principal fator de risco o baixo nível socioeconômico, histórico prévio de depressão e a falta de apoio social à mulher. Importante fator protetor apresentado nos artigos foi a importância da equipe multidisciplinar para o acompanhamento da gestante. A partir do entendimento dos profissionais de saúde acerca dos fatores de risco e proteção da DPP é possível atenuar ou neutralizar o impacto da depressão pós-parto na vida da mulher.

Os artigos selecionados no presente estudo evidenciaram uma relação na utilização de escores para diagnóstico e risco de DPP; determinação de fatores de risco e fatores de proteção para desenvolvimento de DPP, e no tratamento da DPP. Esses pontos colaboram para a identificação precoce de puérperas com risco aumentado de desenvolver depressão ou que já tenham o quadro, possibilitando o manejo eficiente e adequado na Atenção Primária.

No que tange os fatores de risco para desenvolvimento de DPP os quesitos mais prevalentes nos estudos foram: o nível socioeconômico ou nível educacional mais baixo, o histórico de condições prévias semelhantes, a vida marginalizada na sociedade, a experiência pós-traumática, a depressão pré-natal, a anemia, a violência doméstica, a sensação de fadiga e o baixo nível de progesterona. Estes todos foram os fatores significativos para o desenvolvimento de DPP (WASSIF OM, et al., 2019; SHAKEEL N, et al., 2018; MELTZER-BRODY S, et al., 2018; XU F, et al., 2018; TABB KM, et al., 2018; ROGATHI JJ, et al., 2017; GIALLO R, et al., 2015). Os fatores de riscos mais preponderantes encontrados nos estudos foram, no entanto, o baixo nível socioeconômico e educacional da mulher.

No estudo de Oztora S, et al. (2019) os fatores de risco para o desenvolvimento da DPP são: mulheres jovens (principalmente entre 18-25 anos), companheiro(a) desempregado(a), baixa renda mensal, bebê com algum problema de saúde e não aleitamento materno. Já em Xu F, et al. (2018), é constatado que o risco para DPP é aumentado em mulheres com a saúde física precária e aquelas diagnosticadas com anemia. Estudo de Giallo R, et al. (2015) evidencia ainda uma complexa relação bidirecional entre a fadiga e os sintomas depressivos, sem que, necessariamente, a fadiga seja um fator de risco para a DPP. Por exemplo, em mulheres com depressão prévia, espera-se que a depressão tenha vindo antes da fadiga, enquanto em mulheres com filhos subsequentes, a fadiga pode "conduzir" para os sintomas depressivos.

Ainda na perspectiva da depressão prévia, no estudo de Meltzer-Brody S, et al. (2018) revela que mulheres com transtorno mental prévio (mínimo de dois episódios) tiveram maiores riscos de diagnóstico psiquiátrico pós-parto. Além desse, no trabalho de Wassif OM, et al. (2019) houve uma associação significativa entre a história pregressa de condições psiquiátricas semelhantes e a prevalência atual de distúrbios pós-parto. No entanto, a principal limitação destes trabalhos é que, como todos os estudos transversais, eles não podem indicar a causa, mas apenas fatores de associação à depressão pós-parto.

Por fim, Rogathi JJ, et al. (2017) observaram uma forte relação entre mulheres que sofrem algum tipo de violência doméstica e o desenvolvimento de DPP, diferenciando ainda os tipos de violências: física, sexual e emocional. Enfatiza-se que, em todos esses casos, as mulheres vítimas estão mais propensas ao desenvolvimento de depressão devido a situação de vulnerabilidade, se sentindo solitárias, sem amparo e sobrecarregadas emocionalmente.

Há de se considerar que o baixo nível socioeconômico é pauta na maioria dos estudos analisados. Nos artigos de Oztora S, et al. (2019) e Wassif OM, et al. (2019) foram avaliadas características sociodemográficas dos participantes incluindo o seu nível socioeconômico por meio de escalas já validadas, associando assim, de forma significativa, os níveis socioeconômicos mais baixos à DPP.

Ressalta-se ainda que, de acordo com estudo feito por Tabb KM, et al. (2018), mulheres vítimas de violência doméstica têm três vezes mais risco de terem ideação suicida pós-parto, podendo ser considerado um fator de risco mais grave. $\mathrm{Na}$ amostra do estudo $70 \%$ das mulheres que relataram essa ideação suicida foram violentadas. A violência por parceiro íntimo no puerpério compromete a conexão entre mãe e filho por se tratar de um evento estressor traumático. Tal condição apresenta patogenicidade repercutindo nos processos normativos do desenvolvimento infantil. $\mathrm{O}$ artigo ainda salienta sobre a falta de diretrizes práticas sobre a triagem de violência. 
Além disso, Rogathi JJ, et al. (2017) destaca a importância de identificar as mulheres vítimas de violência doméstica a partir de um pré-natal bem feito e com o número adequado de consultas, objetivando um melhor prognóstico. Entretanto, é preciso utilizar instrumentos que possibilitem a identificação precoce e formas mais eficazes de ações diante do problema.

Ainda nessa sobre os fatores de proteção são citados nos estudos acima, no artigo de Xu F, et al. (2018), a depressão pós-parto foi associada à anemia em mulheres antes e após o nascimento, evidenciando, que a prevenção ou tratamento da anemia, possibilitaria prevenir a depressão pós-natal. Um cuidado simples e de fácil manejo, configurando-se de suma importância, principalmente no contexto da atenção primária.

No estudo realizado por $\mathrm{Ma} \mathrm{JH}$, et al. (2019) com 654 mulheres, a quetamina foi identificada como um fator protetor para DPP e fatores de risco associados à DPP. Isso pode ser explicado pelo fato da DPP estar relacionada com a menor concentração de metabólitos de glutamato e pela menor capacidade de resposta do eixo hipotálamo-pituitário-adrenal, com a quetamina capaz de bloquear receptores glutamatérgicos. Assim, a quetamina poderia ser utilizada como uma droga de proteção em mulheres de alto risco em desenvolver DPP, sendo a dose da medicação utilizada no estudo de $0,5 \mathrm{mg} / \mathrm{kg}$ após 10 (dez) minutos do parto. Vale destacar que os efeitos colaterais encontrados neste mesmo estudo com o uso da quetamina foram: vômitos em $19 \%$ dos pacientes, tontura em $5,5 \%$, nistagmo em $3,4 \%$ e alucinação em $1,2 \%$; sendo que o grupo controle apresentou vômitos em 9,4\% da amostra e tonturas em 4,2\% (MA JH, et al., 2019).

Além dos fatores de proteção já citados, a utilização de uma equipe multidisciplinar revelou-se de extrema importância no estudo de Wassif OM, et al. (2019), sendo esse quesito um dos quais pode modificar o prognóstico. A pesquisa corrobora a ideia de que uma combinação de medicamentos, aconselhamento psicológico, grupos de apoio multidisciplinar e estratégias longitudinais são as formas mais eficazes de tratar a DPP (WASSIF OM, et al., 2019).

Por fim, importante ressaltar que, como foi dito anteriormente, segundo Giallo R, et al. (2015), a fadiga foi um sintoma encontrado em $70 \%$ das mulheres diagnosticadas com DPP. Porém, não foi bem estabelecido se é um sintoma presente antes ou após o aparecimento de depressão pós-parto, o que poderia ajudar nas ações de proteção, caso a relação seja de causalidade. O sintoma da fadiga ajuda na identificação da depressão e no manejo, devido ao estigma em torno dos transtornos mentais, já que algumas mulheres se negam a assumir que estão tristes. Tal comportamento é bastante comum, pois socialmente a gravidez e o nascimento de uma criança estão sempre associados a estados de felicidade. Por diversos motivos (culturais, históricos e sociais), ser mãe tornou-se algo compulsório, de modo que muitas mulheres se sentem culpadas por não desejarem um filho ou por, mesmo desejando, terem dúvidas e medos ao tornarem-se mães.

Somado a isso, alguns estudos verificaram as repercussões para a saúde da criança, uma vez que que os bebês que viveram um contexto de depressão materna apresentam apego inseguro, sono irregular, baixa autoestima, menos exploração do ambiente, ansiedade e maior probabilidade de desenvolverem depressão na idade adulta. Isso reforça a importância de se incluir a DPP nas pautas de discussão sobre a saúde mental materna e infantil (MELO SB, et al., 2018).

Outro tópico de importante discussão nos artigos são os escores para o diagnóstico e risco de DPP. Atualmente, a prevalência dessa comorbidade está em torno de $10-15 \%$ no mundo; entretanto, $80 \%$ das mulheres não são diagnosticadas (ROGATHI JJ, et al. 2017). Uma forma de diminuir esse subdiagnóstico é através do escore para diagnóstico e risco de DPP, sendo que a escala mais utilizada ao redor do mundo é a Edinburgh Postnatal Depression Scale (EPDS) (OZTORA S, et al., 2019).

Nessa escala, em que a análise de risco para DPP é verificada, mulheres são solicitadas a dizer, em uma auto avaliação, até que ponto experimentaram uma série de sintomas no período dos últimos sete dias, como por exemplo: sentir-se triste ou infeliz, chorar, sentir-se culpada e olhar para as atividades do cotidiano sem prazer. Ela é, especificamente, uma escala com dez perguntas em que as mulheres avaliam os seus sintomas entre quatro opções de respostas, as quais são valoradas de 1 a 4, dependendo da pergunta. É uma escala considerada ser de fácil aplicação devido a sua linguagem de compreensão simples e sucinta (GIALLO R, et al., 2015). 
Embora essa escala seja utilizada na maior parte dos estudos ao redor do mundo, ainda existe uma pequena divergência em relação ao valor limite considerado para uma possível DPP. Dependendo do país avaliado, se desenvolvido ou em desenvolvimento, alguns estudos consideram como alta probabilidade de DPP valores iguais ou acima de 12, enquanto outros artigos consideram valores iguais ou acima de 13 (OZTORA S, et al., 2019; GIALLO R, et al., 2015). Essa discordância entre os valores de corte pode impactar na análise e resultados dos estudos, visto que, não há um padrão uniforme para se comparar os artigos. Existe assim, esse fator peculiar em se utilizar a EPDS e que deve ser levado em consideração.

Outro tópico importante para a compreensão da DPP são as formas de tratamento. De acordo com Holt C, et al. (2017), mulheres com DPP que foram acolhidas utilizando a técnica de entrevistas motivacionais e usufruindo da ferramenta do cuidado, apresentaram melhora mais rápida e significativa. Em contrapartida, as mulheres também com DPP, mas que receberam como tratamento a abordagem nomeada regular, não apresentaram o mesmo desfecho favorável. Destaca-se que o vínculo entre equipe e paciente, assim como a continuidade de atendimentos, foi maior no primeiro grupo (47\% vs $20 \%$ no segundo grupo).

A Terapia Cognitiva Comportamental (TCC) no período puerperal mostrou ter um efeito positivo na qualidade de vida física e mental das mulheres no pós-parto, principalmente por proporcionar reconstruções cognitivas e habilidades de resolução de problemas. Sendo esse um ponto relevante para a discussão, visto que, muitas mulheres não encontram apoio em seu(a) companheiro(a) ou em outro familiar. A TCC atua também em um dos fatores de risco do desenvolvimento da DPP: a sobrecarga emocional. Com a utilização dessa forma de terapia, é possível proporcionar autoconhecimento à paciente e melhora dos sintomas (NGAI FW, et al., 2017)

É também pertinente apresentar o tratamento medicamentoso utilizado na DPP. Todos os estudos selecionados abordam a TCC como sendo a opção mais adequada de tratamento, evitando-se assim a terapia medicamentosa. No entanto, ao se observar a linha terapêutica proposta pela American Psychiatric Association (APA), é sugerida a utilização de medicação conforme a gravidade do quadro depressivo apresentado, dando preferência ao psicofármaco que a paciente tenha utilizado em algum possível tratamento bem sucedido, no passado (REUS VI, et al, 2016).

Outro fator relevante para a escolha medicamentosa é se a mulher está ou não amamentando. Para mulheres que estão no processo de amamentação é sugerido o uso de inibidores seletivos da recaptação da serotonina (ISRS), especialmente a sertralina e a paroxetina, pois tem menor passagem para o leite (REUS VI, et al., 2016).

Outras opções para essas mulheres são os inibidores da recaptação da serotonina e da noradrenalina (IRSN), como a venlafaxina e a desvenlafaxina. Já para as mulheres que não amamentam, deve-se seguir o mesmo raciocínio de escolha para pacientes adultos com depressão, levando em conta a experiência de uso, perfil de efeitos adversos, disponibilidade na APS e seu custo (REUS VI, et al., 2016).

\section{CONSIDERAÇÕES FINAIS}

A depressão pós-parto é ainda uma doença subdiagnosticada, com aproximadamente $20 \%$ das mulheres com diagnóstico realizado, e além de ser subdiagnosticada também leva certo estigma social, uma vez que há uma ideia enraizada e difundida na sociedade, segundo o qual a maternidade é vista como o destino da mulher, romantizada. Nos artigos avaliados, os possíveis motivos dessa baixa identificação da morbidade não foram apresentados. Sabe-se que o subdiagnóstico é prejudicial para a mãe e para o bebê e com o intuito de ajudar em uma abordagem precoce pode-se lançar mão da utilização da escala EPDS durante o pré-natal e consultas de puerpério. Como apresentado, a baixa condição socioeconômica, o baixo grau de escolaridade e a violência doméstica são os fatores de risco mais prevalentes para DPP; portanto, é essencial pensar em ações sociais e de saúde coletiva que revertam, minimamente, esse contexto. Já o tratamento deve contar com uma equipe multidisciplinar, que permita acolher e escutar a paciente, não se restringindo apenas ao uso de medicamentos antidepressivos. 


\section{REFERÊNCIAS}

1. ARRAIAS AR, ARAUJO TCCF. Depressão pós parto: uma revisão sobre fatores de risco e proteção. Psicologia, Saúde \& Doenças, 2017; 18(3): 828-845.

2. Comitê de Prática Obstétrica. Parecer $\mathrm{n} \times 630$ do Comitê do Colégio Americano de Obstetras e Ginecologistas: triagem para depressão perinatal. ObstetGynecol. 2015; 125 5): 1268-1271.

3. FERREIRA C, et al. Postpartum depression: early detection and associated factors. Acta Obstet Ginecol Port, 2018; 12(4): 262-267

4. Força-Tarefa de Serviços Preventivos dos EUA. Intervenções para prevenir a depressão perinatal: Declaração de recomendação da Força-Tarefa de Serviços Preventivos dos EUA. JAMA. 2019; 321(6): 580-587.

5. GIALLO R, et al. "I didn'tknow it was possible to feel that tired": exploring the complex bidirectional associations between maternal depressive symptoms and fatigue in a prospective pregnancy cohort study. Arch Womens Ment Health. 2016; 19(1): 25-34.

6. HARRIS L. The Lancet. Screening for perinatal depression: a missed opportunity [editorial]. Lancet. 2016; 387(10018): 505.

7. HOLT C, et al. Improving help-seeking for postnatal depression and anxiety: a cluster randomised controlled trial of motivational interviewing. Arch Womens Ment Health. 2017; 20(6): 791-801.

8. KO JY, et al. Tendências dos sintomas depressivos pós-parto - 27 estados, 2004, 2008 e 2012. MMWR Morb Mortal WklyRep. 2017; 66(6): 153-158.

9. $\mathrm{MA} \mathrm{JH}$, et al. Prophylactic use of ketamine reduce postpartum depression in Chinese women undergoing cesarean section. PsychiatryResearch. 2019; 279: 252-258.

10. MELO SB, et al. Sintomas depressivos em puérperas atendidas em Unidades de Saúde da Família. Rev. Bras. Saúde Mater. Infant. 2018; 18(1): 171-177.

11. MELTZER-BRODY S, et al. Adverse life events increased risk for postpartum psychiatric episodes: A populationbased epidemiologic study. DepressAnxiety. 2018; 35(2): 160-167.

12. MUNK-OLSEN T, et al. Use of primary health care prior to a postpartum psychiatric episode. Scand J Prim Health Care. 2015; 33(2): 127-133.

13. NGAI FW, et al. The effect of a telephone-based cognitive behavioraltherapyon quality of life: a randomized controlled trial. ArchWomensMent Health. 2017;20(3):421-426.

14. OZTORA S, et al. Postpartum Depression And Affecting Factors in primary care. Niger J ClinPract. 2019; 22(1): 8591.

15. O'CONNOR E, et al. Primary Care Screening for and Treatment of Depression in Pregnant Postpartum Women: Evidence Reporting Systematic Review for the US Preventive Services Task Force. JAMA. 2016; 315(4): 388-406.

16. REUS VI, et al. The American Psychiatric Association Practice Guideline on the Use of Antipsychotics. Am J Psychiatry. 2016;173(5):543-546.

17. ROGATHI JJ, et al. Postpartum depression among women who have experienced intimate partner violence: $A$ prospectivecohortstudyatMoshi, Tanzania. J Affect Disord. 2017; 218: 238-245.

18. SHAKEEL N, et al. Prevalence Postpartum Depressive Symptoms in a multiethnic population andthe role of ethnicity and integration. J Affect Disord. 2018; 241: 49-58.

19. TABB, KM, et al. Intimate Partner Violence Is Associated with Suicidality Among Low-Income Postpartum Women. Journal of Women's Health. 2018; 27(2): 171-178.

20. US Preventive Services Task Force. InterventionstoPrevent Perinatal Depression: US Preventive Services Task Force RecommendationStatement. JAMA. 2019; 321(6): 580-587.

21. WASSIF OM, et al. Assessment of Postpartum Depression Health Care Facilities in Qalyubia Governorate, Egypt. J EnvironPublic Health. 2019;2019:3691752.

22. XU F, et al. Anaemia And Depression Before And After Birth: a cohort study based on linked population data. BMC Psychiatry. 2018; 18(1): 224. 